\title{
Daxx and TCF4 interaction links to oral squamous cell carcinoma growth by promoting cell cycle progression via induction of cyclin D1 expression
}

\author{
Gu-Jiun Lin ${ }^{1}$ • Yen-Sung Huang ${ }^{2}$ - Chih-Kung Lin ${ }^{3}$ - Shing-Hwa Huang ${ }^{1,4}$. \\ Hsiu-Ming Shih ${ }^{2} \cdot$ Huey-Kang Sytwu ${ }^{5}$ Yuan-Wu Chen ${ }^{6,7}$
}

Received: 12 May 2015 / Accepted: 13 July 2015 /Published online: 24 July 2015

(C) The Author(s) 2015. This article is published with open access at Springerlink.com

\begin{abstract}
Objectives Death domain-associated protein (Daxx) has been recently implicated as a positive factor in ovarian cancer and prostate cancer, but the role of Daxx in oral squamous cell carcinoma (OSCC) has never been addressed. Herein, we investigate the expression and function of Daxx in OSCC.

Materials and methods RT-quantitative PCR, Western blotting, and immunohistochemistry were used to evaluation of the expression of Daxx in human OSCC cell lines and clinical surgical specimens. Short hairpin RNA targeting Daxx was transduced by lentivirus infection to knockdown the expression of Daxx in SAS and SCC25 cell lines, and the influence of this knockdown was evaluated by analyzing the growth and the cell cycle in transduced cells. Immunoprecipitation and sequential chromatin immunoprecipitationquantitative PCR were used to analyze the associations
\end{abstract}

Gu-Jiun Lin and Yen-Sung Huang contributed equally to this work.

Yuan-Wu Chen

H6183@yahoo.com.tw

1 Department of Biology and Anatomy, National Defense Medical Center, Taipei, Taiwan

2 Institute of Biomedical Sciences, Academia Sinica, Taipei, Taiwan

3 Department of Pathology, Taipei Tzu Chi general Hospital, New Taipei City, Taiwan

4 Department of General Surgery, Tri-Service General Hospital, National Defense Medical Center, Taipei, Taiwan

5 Department and Graduate Institute of Microbiology and Immunology, National Defense Medical Center, Taipei, Taiwan

6 Department of Oral and Maxillofacial Surgery, Tri-Service General Hospital, Taipei, Taiwan

7 School of Dentistry, National Defense Medical Center, 325 Cheng-Kung Road, Section 2, Nei-Hu, Taipei 11490, Taiwan between Daxx, TCF4, and cyclin D1 promoter. Xenograft tumor model was used to evaluate the in vivo tumorigenicity of Daxx in OSCC.

Results Daxx mRNA and protein expression are elevated in several OSCC cell lines and human OSCC samples in comparison to those in normal tissue. We further find that depletion of Daxx decreases OSCC cell growth activity through G1 cell cycle arrest. Daxx silencing reduces cyclin D1 expression via a Daxx-TCF4 interaction, whereas the Daxx depletionmediated G1 arrest can be relieved by ectopic expression of cyclin D1. Moreover, we show that in OSCC clinical samples, the expression of Daxx is significantly correlated with that of cyclin D1.

Conclusion Our data demonstrate the importance of Daxx in regulation of cyclin D1 expression and provide the first evidence that Daxx exhibits tumor-promoting activity in OSCC. Clinical relevance Daxx plays an important role in malignant transformation of OSCC and may serves as a target for cancer prevention and treatment.

Keywords OSCC $\cdot$ Daxx $\cdot$ TCF $4 \cdot$ Cyclin D1 $\cdot$ Cell cycle

\section{Introduction}

Head and neck squamous cell carcinomas, including oral squamous cell carcinomas (OSCC), are the eighth most prevalent new cancer cases among men in the USA in 2014 [1]. The clinical outcome and prognosis for OSCC are dismal; more than $61 \%$ of patients die of this disease or its complications within 5 years [2]. Thus, identifying cancer relative molecular markers for clinical histopathologic exams and finding the regulatory mechanisms of tumorigenicity in OSCC are necessary for development of potential therapeutics. 
Daxx is a predominant nuclear protein that associates with several different subnuclear structures, including the PML nuclear body, heterochromatin, and nucleolus [3]. There are numerous reported functions for Daxx, acting as apoptosis regulator and transcription co-regulator in a SUMO bindingdependent or binding-independent manner [3-5]. Moreover, cooperation of Daxx with the chromatin remodeler ATRX is required for histone H3.3 deposition at the pericentromeric and telomere regions; H3.3 deposition facilitates transcript expression from pericentromeric regions [6]. Analysis derived from clinical samples in pancreatic neuroendocrine tumors (PanNETs) revealed a high ratio of inactivating-to-missense mutations in the coding region of Daxx [7]. Daxx has been recently implicated as a tumor promoting factor in ovarian cancer and prostate cancer, but the role of Daxx in OSCC has never been addressed $[8,9]$.

Although several Daxx-interacting proteins are involved in critical cellular pathways regarding P53 degradation, such as ubiquitin-specific-processing protease 7 (USP7) and mouse double minute 2 (Mdm2) [10, 11], it is still unclear whether Daxx has any role in tumorigenicity of OSCC. In the present study, we investigated the expression and the function of Daxx in OSCC. Our results provide the first evidence of elevated Daxx expression in both OSCC cell lines and clinical samples. We further demonstrated that Daxx silencing by RNAi reduces the cell growth of OSCC in vitro and in vivo. Daxx silencing reduces cyclin D1 expression and increases the fraction of cells in the G1 phase in OSCC, whereas ectopic expression of cyclin D1 eliminates the Daxx depletion-induced G1 cell cycle arrest. Together, our results suggest that Daxx silencing reduces OSCC cell growth through cyclin D1 downregulation.

\section{Materials and methods}

\section{Tissue samples and ethics statement}

All OSCC and matched normal tissues were obtained from 18 OSCC patients in the Department of Oral and Maxillofacial Surgery at Tri-Service General Hospital, Taiwan. The clinical and histological characteristics of the surgical specimens were presented in an additional Table 1. The experiments were under-taken following the approval by the Ethical Committee of Tri-Service General Hospital IRB protocol 096-05-002-I and 02-101-05-082, and written informed consents were obtained from all patients. The animal experiments were under-taken following the approval by the Institutional Animal Care and Use Committee of National Defense Medical Center IACUC protocol 11-084. All efforts were made to minimize the suffering of experimental animals.
Table 1 Clinical and histological characteristics of the 25 cases of primary human oral squamous cell carcinoma used in immunohistochemical analysis

\begin{tabular}{|c|c|c|c|c|c|}
\hline Case No. & Gender & Age & Anatomic site & Stage & Histological grade \\
\hline 1 & Male & 63 & Anterior pillar & I & Moderate \\
\hline 2 & Male & 37 & Buccal & IVa & Poor \\
\hline 3 & Male & 44 & Buccal & III & Poor \\
\hline 4 & Male & 66 & Buccal & III & Moderate \\
\hline 5 & Male & 47 & Buccal & IVa & Moderate \\
\hline 6 & Male & 44 & Buccal & IVa & Moderate \\
\hline 7 & Male & 52 & Buccal & IVa & Poor \\
\hline 8 & Male & 60 & Gingiva & IVa & Moderate \\
\hline 9 & Female & 42 & Gingiva & I & Moderate \\
\hline 10 & Male & 82 & Gingiva & I & Moderate \\
\hline 11 & Female & 75 & Gingiva & IVa & Well \\
\hline 12 & Male & 71 & Lip & II & Moderate \\
\hline 13 & Male & 52 & Mandible & $\mathrm{IVb}$ & Moderate \\
\hline 14 & Male & 57 & Mouth floor & IVa & Poor \\
\hline 15 & Male & 39 & Mucosa & IVa & Moderate \\
\hline 16 & Male & 48 & Oral cavity & III & Poor \\
\hline 17 & Male & 45 & Soft palate & III & Moderate \\
\hline 18 & Male & 40 & Tongue & I & Well \\
\hline 19 & Male & 47 & Tongue & IVa & Moderate \\
\hline 20 & Male & 44 & Tongue & IVa & Poor \\
\hline 21 & Male & 52 & Tongue & I & Moderate \\
\hline 22 & Male & 70 & Tongue & I & Moderate \\
\hline 23 & Female & 74 & Tongue & III & Moderate \\
\hline 24 & Male & 47 & Tongue & II & Moderate \\
\hline 25 & Female & 48 & Tongue & II & Poor \\
\hline
\end{tabular}

\section{Cells, siRNA, plasmids, and transfection}

293T, WI38, and SCC25 cell lines were obtained from the American Type Culture Collection. The 293T and SCC25 cell lines used have been previously described in detail $[5,12]$. Cell line WI38 was maintained in a minimal amount of essential medium supplemented with $10 \%$ fetal bovine serum (FBS, non-essential amino acids, $1 \mathrm{mM}$ sodium pyruvate, $2 \mathrm{mM}$ L-glutamine, and antibiotics). The SAS cell line was provided by Dr. Jeng-Fan Lo and was grown in high glucose DMEM with $10 \%$ FBS, as described [13]. The specific siRNA oligonucleotides used against Daxx and for negative controls were synthesized by Life Technologies [14]. The lentivirus plasmid expressing shLuc and shDaxx were obtained from the RNAi consortium at Academia Sinica. The shDaxx nucleotide sequences corresponded to Daxx coding sequence 1585-1605. Cyclin D1 plasmid was donated by Dr. William Hahn (Addgene plasmid no. 9050). Plasmids were isolated using a GenElute HP EndoFree Plasmid Maxiprep Kit (Sigma, St. Louis, MO, USA), and transfection was 
performed with a PolyJet (SignaGen Laboratories Ijamsville, MD, USA), according to the manufacturer's instructions.

\section{Immunoprecipitation and Western blotting assays}

Cells were lysed directly in an RIPA buffer containing $50 \mathrm{mM}$ Tris ( $\mathrm{pH} 7.8$ ), $0.15 \mathrm{M} \mathrm{NaCl}, 5 \mathrm{mM}$ EDTA, $0.5 \%$ Triton X-100, $0.5 \%$ NP-40, $0.1 \%$ sodium deoxycholate, and protease inhibitor mixture (Sigma). The relative protein concentration in the supernatants was determined using a BCA protein assay kit (Thermo Scientific, Rockford, USA). For immunoprecipitation, $400 \mu \mathrm{g}$ of protein lysates were incubated with specific antibodies. For each lane of 8 to $10 \%$ SDS-PAGE gel, $40 \mu \mathrm{g}$ protein of cell lysates were loaded, separated, and transferred onto polyvinyldifluoride (PVDF) membrane (Millipore, Bedford, MA, USA). The membranes were then probed using specific antibodies against Daxx (Sigma, D7810), cyclin D1 (Santa Cruz Biotech, Santa Cruz, CA, USA, sc-718), and $\beta$-actin (Sigma, A5441).

\section{RT-quantitative PCR}

Total RNA was extracted from these cells using TRIzol (Life Technologies, Carlsbad, CA, USA) or Total RNA Miniprep Purification kit (GeneMark, Taipei, Taiwan). Five micrograms of RNA from each sample were then reverse transcribed using Superscript III Reverse Transcriptase (Life Technologies). RTquantitative PCR (RT-qPCR) was performed using SYBR Green PCR master mix (Life Technologies) and an ABI 7500 sequence detection system (Life Technologies). The RT-qPCR primers used were as follows: Daxx forward, 5'TGC AGA CAC CCC CGA AGC CT-3'; Daxx reverse primer, 5'-TGC CAT TCC ACT AGG GCC CTC A-3'; GAPDH forward, 5'-TCT TTT GCG TCG CCA GCC GAG-3'; GAPDH reverse primer, 5'-TGA CCA GGC GCC CAA TAC GAC-3'; cyclin D1 forward primer, 5'-TGT GAC CCG GAC TGC CTC CG-3'; and cyclin D1 reverse primer, 5'-GCG CAG GCT TGA CTC CAG CA-3'.

\section{Cell cycle analysis}

Infected cells and transfected cells were harvested for the indicated periods, washed with ice-cold PBS, fixed overnight with $70 \%$ ethanol at $4{ }^{\circ} \mathrm{C}$, and then suspended in $500 \mu \mathrm{L}$ PBS. After adding $10 \mu \mathrm{L}$ RNase A (Sigma, $10 \mathrm{mg} / \mathrm{mL}$ ), cells were allowed to stand at $37{ }^{\circ} \mathrm{C}$ for $30 \mathrm{~min}$, stained with $10 \mu \mathrm{L}$ 7AAD (Sigma, $1 \mathrm{mg} / \mathrm{mL}$ ), and then analyzed by flow cytometry. Flow cytometric analysis was performed using FACSCaliber (BD Biosciences, San Jose, CA, USA), and the data were analyzed using CellQuest software (BD Biosciences).

\section{Lentivirus production and infection}

The replication deficient lentivirus was prepared by transfecting 293T cells with plasmids using a PolyJet (SignaGen Laboratories). Viral supernatants were harvested $72 \mathrm{~h}$ after transfection, clarified through centrifugation, filtered, and stored in aliquots at $-80{ }^{\circ} \mathrm{C}$. Cells were infected by lentivirus supernatants in the presence of $7.5 \mu \mathrm{g} / \mathrm{mL}$ Polybrene (Sigma) for $24 \mathrm{~h}$.

\section{Xenograft tumor model}

Eight-week-old NOD.CB17 Prkdc $c^{\text {scid } / J}$ (National Laboratory Animal Center, Taiwan) mice were maintained in microisolator in pathogen free conditions. Five mice per group, which were randomly assigned, were injected subcutaneously with either shLuc or shDaxx lentivirus infected SAS cells. The sizes of the transplanted tumors were measured with gauged calipers every 3 days, and the tumor volumes were calculated using the following formula: $V=1 / 2 \times($ length $\times$ width ${ }^{2}$ ). At the end of treatment, the mice were euthanized, and the tumors were removed, weighed, and photographed.

\section{Immunohistochemistry}

Parraffin sections were deparaffinized with xylene and rehydrated with a serial grade of alcohol. Epitope retrieval was carried out in a $10-\mathrm{mM}$ citrate buffer $(\mathrm{pH} 6.0)$ water bathed at $90^{\circ} \mathrm{C}$ on a hot plate for $18 \mathrm{~min}$. After inactivation of endogenous peroxidase with $\mathrm{H}_{2} \mathrm{O}_{2}$, these slides were then incubated with specific antibodies against Daxx (Sigma, D7810) or cyclin D1 (Sigma, HPA027801) for $1 \mathrm{~h}$ at room temperature. Immunostaining was performed according to standard procedures. Envision plus kit (DAKO, Carpinteria, CA, USA) was used as a secondary reagent. Stainings were developed using DAB (brown precipitate). Slides were counterstained with hematoxylin and visualized by light microscopy. All immunostains were evaluated by two pathologists. Staining percentage was scored by counting the numbers of staining positive and calculating the percentage of positive cells. The scores of staining percentage was defined as 0 (0 \%), 1 (1-25\%), 2 (26-50\%), 3 (51-75\%), and 4 (76$100 \%)$. Staining intensity was determined by estimating the signal density and scored as 0 (no detectable stain), 1 (weak staining detected at intermediate to high power), 2 (moderate detected at low to intermediate power), to 3 (strong detected at low power). The final immunoreactivity score was scored by multiplying the staining percentage of positive cells by the staining intensity. Sample scores of 7-12 were defined as having high immunoreactivity, and sample scores of 0-6 were defined as having low immunoreactivity. 


\section{Sequential chromatin immunoprecipitation-quantitative PCR assay}

Chromatin immunoprecipitation-quantitative PCR (ChIPqPCR) was performed as previously described [5]. ChIPqPCR product was analyzed by quantitative real-time PCR using the Applied Biosystem 7500 Real-Time PCR System. A fraction (1\%) of the sonicated chromatin was set aside as input control before antibody affinity manipulations. Percent input was calculated by $100 \times 2\left(\mathrm{Ct}^{\text {adiusted Input }-\mathrm{Ct}^{\mathrm{IP}}}\right)$. Primers used to amplify DNA fragments containing the TCF4 consensus site were 5'-AGG CGC GGC GGC TCA GGG ATG-3' and 5'-ACT CTG CTG CTC GCT GCT ACT-3' for the human cyclin D1 promoter [15].

\section{Statistical analysis}

All analyses performed with SigmaPlot software. The twotailed Students's $t$ test was used to evaluate the significance of the differences between two groups of data in all experiments. Values of $P<0.05$ were considered significant.

\section{Results}

Daxx expression is frequently upregulated in both OSCC human samples and cell lines

To examine whether Daxx expression were dysregulated in human OSCC samples, we measured the Daxx mRNA levels in 18 pairs of OSCC tissues and their matched normal mucosa tissues. Among the 18 patients, 14 (78\%) patients showed higher Daxx RNA level in the OSCC tissues than that in adjacent normal mucosa tissues (Fig. 1a). We further analyzed Daxx protein in a set of human OSCC samples and observed that Daxx expression was significantly higher in OSCC tissues than in normal mucosa tissues (Fig. 1b). Notably, Daxx was located in nuclei of normal mucosa tissue and OSCC tissue (Fig. 1c). The results indicated that higher staining intensity and percentage of Daxx positive cells in tumor tissue compared to normal tissue (Fig. 1c). We also investigated the expression levels of Daxx in OSCC cell lines (SCC25 and SAS) and a normal cell line (WI38). SCC25 and SAS cell lines expressed higher levels of Daxx than WI38 cell line (Fig. 1d). Taken together, Daxx expression is frequently upregulated in both OSCC clinical samples and OSCC cell lines.

\section{Downregulation of Daxx reduces the growth of OSCC cells in vitro and in vivo}

The elevated Daxx expression in both OSCC cell lines and OSCC clinical samples led us to investigate whether endogenous Daxx affects the growth capability of OSCC cell. Lentivirus-mediated shRNA was used to knockdown the Daxx protein expression in both SCC25 and SAS OSCC cell lines. This shDaxx plasmid has been widely used to target Daxx mRNA in several studies $[5,16]$. The results indicated that effective silencing of Daxx expression in SCC25 and SAS cells significantly reduces the cell number compared to control cells at day 3 after infection (Fig. 2a, b). Another siRNA oligo against Daxx in a different coding region was also used. Consistent with the lentivirus results, Daxx siRNA (siDaxx) decreases SAS cell viability compared to control cells at day 3 after transfection (Fig. 2c). Xenograft tumor experiments were used to extend the study of endogenous Daxx in tumorigenicity. The results indicated that shDaxx-infected cells significantly reduce tumor growth rates in NOD/SCID mice compared with shLuc-infected cells (Fig. 2d). Collectively, these results further support the notion that suppression of Daxx expression significantly decreases the tumor growth of OSCC.

\section{Daxx silencing reduces cyclin D1 expression via a Daxx-TCF4 interaction}

It has been previously reported that Daxx regulates the expression of cyclin D1 at the transcription level in colorectal cancer [17] and that cyclin D1 is important for OSCC growth activity [18]; we therefore tested whether Daxx can regulate cyclin D1 expression in OSCC using OSCC cell line. We found that suppression of Daxx by RNAi decreases the mRNA transcription and protein expression of cyclin D1 in SAS cells, an OSCC cell line (Fig. 3a). Previous studies indicated that expression of cyclin D1 is strongly dependent on $\beta$-catenin/ TCF4-mediated transcriptional regulation [19]. We showed an endogenous interaction between Daxx and TCF4 in SAS cells using immunoprecipitation (IP) followed by Western blotting (Fig. 3b, left panel). Moreover, the association of Daxx and TCF4 was found on endogenous cyclin D1 promoter in SAS cells as evidenced by the presence of the TCF4-containing DNA fragment precipitated by anti-TCF4 antibody followed by sequential chromatin immunoprecipitation (ChIP) with antiDaxx antibody but not by a control antibody (Fig. 3b, right panel). As we hypothesized that transcription of cyclin D1 is regulated by Daxx, we therefore examined whether cyclin D1 is coexpressed with Daxx in human OSCC samples. We evaluated Daxx and cyclin D1 expression in OSCC clinical samples by RT-qPCR and found a strong correlation between Daxx and cyclin D1 expression ( $r=0.7074, P=0.0149$, data not shown). Similar results were also observed from immunohistochemistry (Fig. 3c, $n=25$ ). Accordingly, Daxx expression 
Fig. 1 Daxx expression is frequently upregulated in OSCC clinical samples and OSCC cell lines. a Quantitative RT-PCR results from 18 pairs of matched normal mucosa tissue and OSCC tissues. b Immunohistochemitry of Daxx expression in a normal mucosa tissue and OSCC tissue. Statistical significance was ascertained by conducting Student's $t$ tests. c Positive nuclear immunolabeling of Daxx in normal mucosa tissue and OSCC tissue. Scale bar $=200$ um. d Western blots of Daxx expression in a normal cell line (WI38) and two human OSCC cell lines (SCC25 and SAS). $\beta$ actin was used as a loading control a

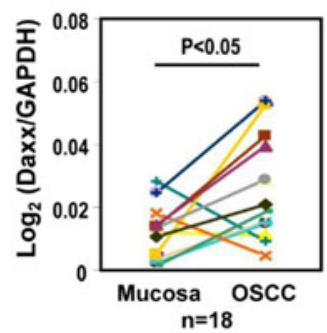

b

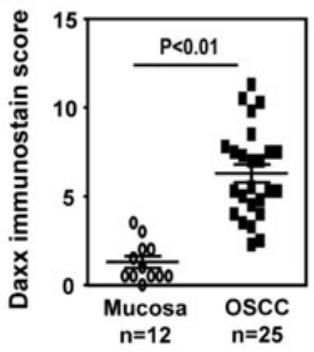

d

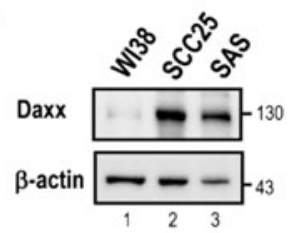

C

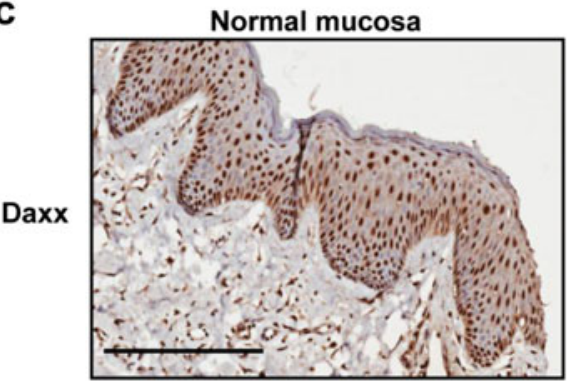

oscc

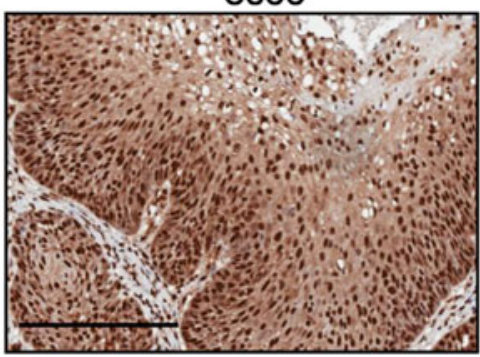

level was correlated with cyclin D1 level in different OSCC samples (Fig. 3d). Together, our results suggest that Daxx functions as a positive regulator in modulating cyclin D1 expression via interacting with TCF4 in OSCC cells.
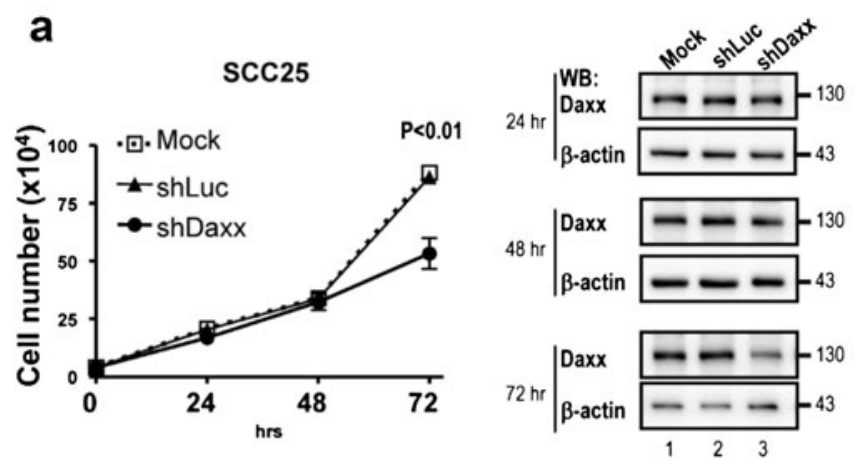

C

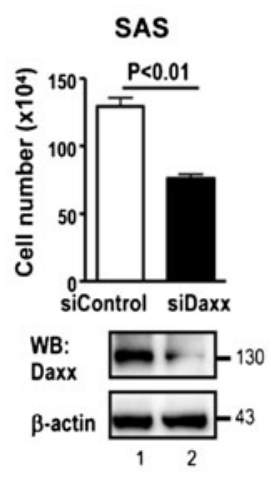

d

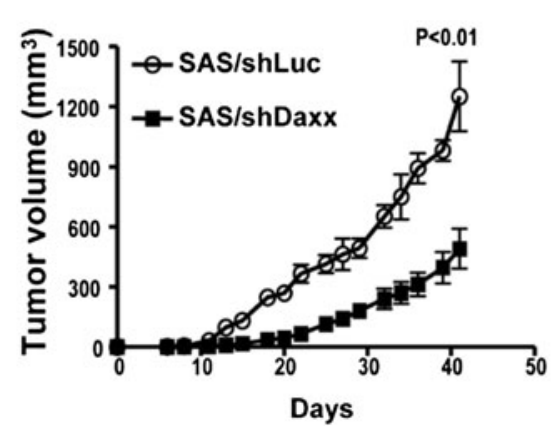

b

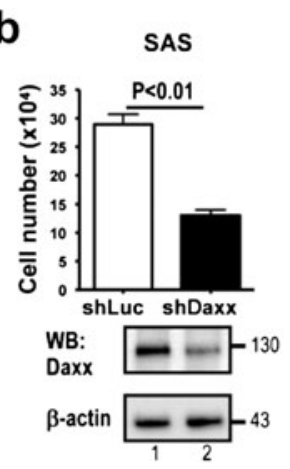

Fig. 2 RNAi-mediated down-regulation of Daxx reduces the growth activity of OSCC cells in vitro and in vivo. a SCC25 and b SAS cells infected with shLuc and shDaxx lentivirus were counted at the indicated times. Infected cells were then subjected to Western blots with indicated antibodies. c SAS cells transfected with siControl and siDaxx were counted at day 3 after transfection and subjected to Western blots with indicated antibodies. d SAS cells infected with shLuc and shDaxx were subcutaneously xenografted into NOD/SCID mice ( $n=5$ mice per group). Data are represented as mean \pm SEM. The tumor size was analyzed using Student's $t$ test at day 42 after tumor cell injection (left panel). Top right panel: Western blot of an aliquot of the infected cells before injection. Bottom right panel: tumors from SAS/shLuc or SAS/shDaxx were excised at day 42 

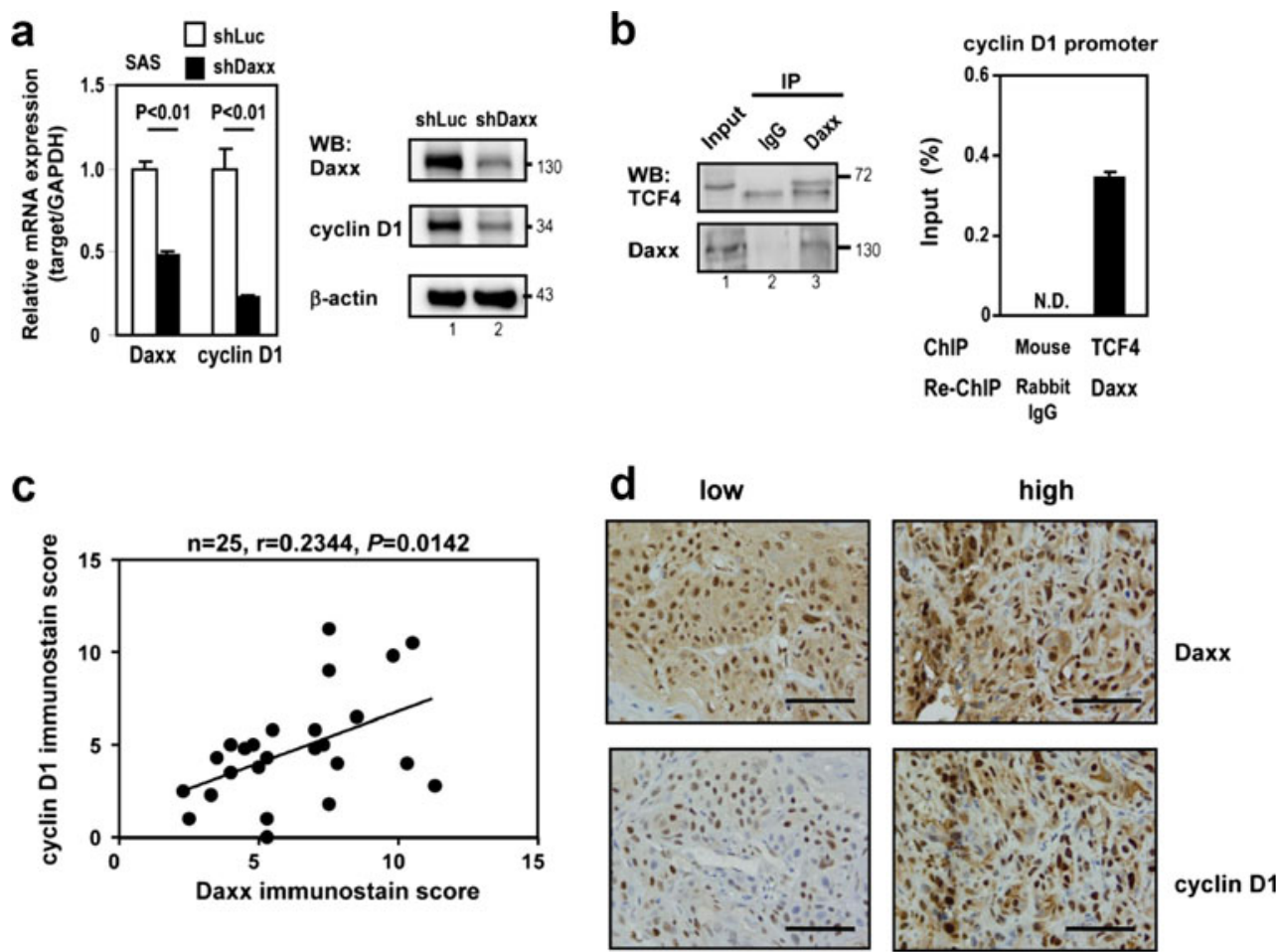

Fig. 3 Daxx silencing reduces cyclin D1 expression via a Daxx-TCF4 interaction. a RT-qPCR and Western blot of cyclin D1 expression in Daxx shRNA-infected SAS cells. Data are presented as the mean \pm SD from three experiments. Statistical significance was ascertained using Student's $t$ test. b Western blot and sequential chromatin immunoprecipitation (ChIP) assays were performed with antibodies against Daxx, TCF4, or IgG control. Input represents $5 \%$ of the lysates used for Western blot and $1 \%$ of the chromatin used for ChIP,

respectively. Data are presented as the mean $\pm \mathrm{SD}$ from three independent experiments. N.D. not detected. c A positive correlation between Daxx ( $x$ axis) and cyclin D1 ( $y$ axis) in OSCC samples using immunohistochemistry. Each dot corresponds to one sample. Statistical significance was ascertained using Regression analysis. $\mathbf{d}$ Representative photographs of Daxx- and cyclin D1-stained tumors collected from OSCC clinical samples with low and high staining

\section{Daxx silencing induces cyclin D1-mediated G1 arrest in OSCC cells}

Given that cyclin D1 plays a critical role in the G1 to S phase cell cycle transition [19], we expect that Daxx silencing should induce G1 arrest. We therefore examined the effects of Daxx silencing on cell-cycle progression. Indeed, suppressing Daxx in SCC25 and SAS cells enhanced the portion of cells in the G1 phase (Figs. 4a, b). To confirm this notion, cyclin D1 was ectopically expressed in Daxx-depleted SAS cells. Our results showed that ectopic expression of cyclin D1 eliminates Daxx depletion-induced G1 arrest (Fig. 4c). Taken together, Daxx regulates the growth activity of OSCC cells which may be associated with cyclin D1.

\section{Discussion}

Aberration of cell cycle regulation accounts for many abnormal biological behavior of cancer cells. In this study, we show that Daxx silencing in OSCC cells increases the number of G1-arrested cells through downregulating cyclin D1 expression. Cyclin D1 is a vital protein that controls the transition

from $\mathrm{G} 1$ to $\mathrm{S}$ phase and governs the rate of cell proliferation. Increase or upregulation of cyclin D1 has been reported in about 36 to $56 \%$ of OSCC patients, and therefore, it acts as an important genetic event in OSCC [18]. Recently, Zhao et al. [20] indicated that cyclin D1 expression correlates with detrimental clinicopathological outcome and poor prognosis in OSCC. Thus, it is possible that Daxx may be used as a prognostic factor for OSCC. In the current study, we provide evidence for a possible link between Daxx expression level and the pathogenesis of OSCC. How the correlation between Daxx and cyclin D1 is involved in clinicopathological features and poor prognosis of OSCC needs to be dissected in the future.

In this current study, we have shown that Daxx silencing reduces tumor growth by suppressing cyclin D1 expression in OSCC. In line with this notion, a few recent studies indicate that Daxx may play a tumor promoting role in several cancer types. For example, Daxx destabilizes P53 via inhibition of Mdm2 ubiquitination in osteosarcoma and colorectal cancer cells [10]. In addition, Daxx potentiates TCF4/ $\beta$-catenin-mediated transcriptional activation in colorectal cancer cells [17]. Furthermore, Daxx is frequently overexpressed in prostate cancer cells and the expression level is significantly correlated 

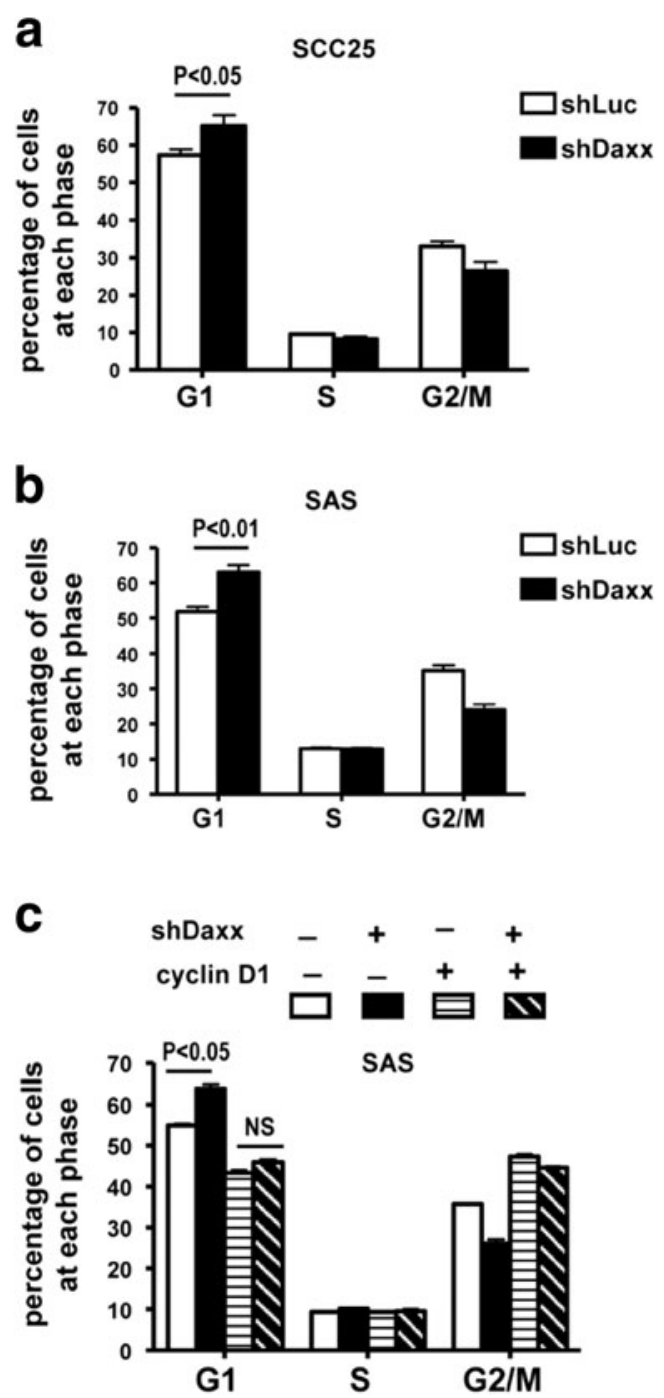

Fig. 4 Daxx silencing induces cyclin D1-mediated G1 arrest in OSCC cells. a SCC25 cells and b SAS cells infected with lentivirus containing shLuc or shDaxx for 3 days were fixed and stained with 7-AAD for cell cycle analysis. c Flow cytometric cell cycle analysis of SAS cells transfected with plasmids as indicated after 3 day. Data are presented as the mean \pm SD from three independent experiments. Statistical significance was ascertained by conducting Student's $t$ tests

with prostate cancer stage [8,21]. Moreover, Daxx silencing suppresses the growth activity of human ovarian cancer cells and mouse ovarian surface epithelial cells $[9,22]$. Development of OSCC is a multi-step progressing process of transformation from healthy mucosa to invasive carcinoma. Each step is accompanied by specific genetic alterations, including inactivation of P53 and overexpression of cyclin D1 [23]. Since Daxx is a negative regulator of P53 and a positive regulator of cyclin D1, these facts support the notion that Daxx exerts a tumor promoting effect in OSCC or other types of cancer. However, these results are derived from cell models. It is important to explore the oncogenic contribution of Daxx in different cancer models using conditional gene knockout mice.
This is the first study to demonstrate the underlying mechanism in OSCC of how the expression of cyclin D1 is regulated by Daxx through an interaction with TCF4 on the cyclin D1 promoter. Even though the molecular basis of how Daxx enhances cyclin D1 expression is currently unclear, we speculate two possible scenarios. First, Daxx may recruit CBP to potentiate TCF4-mediated transactivation since Daxx could function as a coactivator through recruiting CBP in a cell-contextdependent manner [24]. Secondly, Daxx acts as a chaperone for the histone variant $\mathrm{H} 3.3$ which is enriched at centromeric heterochromatin, telomeres, and active genes. Therefore, Daxx may regulate transcription through loading $\mathrm{H} 3.3$ at regulatory regions [25]. These two possibilities are not mutually exclusive. Further studies are required to clarify the molecular mechanism of Daxx in enhancement of cyclin D1 expression.

In sum, we have discovered for the first time that the expression of Daxx mRNA and protein was upregulated in OSCC clinical samples as well as in OSCC cell lines. Moreover, knocking down Daxx inhibited cell growth through inducing G1 cell cycle arrest and decreased cyclin D1 expression. Hence, Daxx silencing may reduce tumorigenicity in OSCC. Thus, it is reasonable to speculate that Daxx plays an important role in malignant transformation of OSCC and serves as a potential target for cancer prevention and treatment. Further mechanistic studies may advance our knowledge of the role of Daxx in OSCC and cancer development.

Acknowledgments We thank Dr. Jeng-Fan Lo (Institute of Oral Biology, School of Dentistry, National Yang-Ming University) for providing SAS cells and Dr. William C. Hahn (Dana-Farber Cancer Institute) for providing cyclin D1 plasmid, respectively. We are grateful to Chin-Hsiu Tseng for comments on the paper. This study was supported by research grants from Tri-Service General Hospital, Republic of China (grants no. TSGH-C103-005-007-009-S06 and TSGH-C104-008-S05), Ministry of National Defense, Taiwan, Republic of China (grants no. 103-M055 and 103-M075), and Ministry of Science and Technology, Taiwan, Republic of China (grants no. NSC102-2314-B-016-018-MY3 to Y.-W. Chen and MOST 103-2321-B-016-008 to G.-J. Lin). The funders had no role in study design, data collection and analysis, decision to publish, or preparation of the manuscript.

Conflict of interest The authors declare that they have no competing interests.

Open Access This article is distributed under the terms of the Creative Commons Attribution 4.0 International License (http:// creativecommons.org/licenses/by/4.0/), which permits unrestricted use, distribution, and reproduction in any medium, provided you give appropriate credit to the original author(s) and the source, provide a link to the Creative Commons license, and indicate if changes were made.

\section{References}

1. Siegel R, Desantis C, Jemal A (2014) Colorectal cancer statistics, 2014. CA Cancer J Clin 64:104-117

2. Liu SY, Lu CL, Chiou CT, Yen CY, Liaw GA, Chen YC, Liu YC, Chiang WF (2010) Surgical outcomes and prognostic factors of oral 
cancer associated with betel quid chewing and tobacco smoking in Taiwan. Oral Oncol 46:276-282

3. Salomoni P, Khelifi AF (2006) Daxx: death or survival protein? Trends Cell Biol 16:97-104

4. Shih HM, Chang CC, Kuo HY, Lin DY (2007) Daxx mediates SUMO-dependent transcriptional control and subnuclear compartmentalization. Biochem Soc Trans 35:1397-1400

5. Huang YS, Chang CC, Huang TC, Hsieh YL, Shih HM (2012) Daxx interacts with and modulates the activity of CREB. Cell Cycle 11:99-108

6. Goldberg AD, Banaszynski LA, Noh KM, Lewis PW, Elsaesser SJ, Stadler S, Dewell S, Law M, Guo X, Li X, Wen D, Chapgier A, DeKelver RC, Miller JC, Lee YL, Boydston EA, Holmes MC, Gregory PD, Greally JM, Rafii S, Yang C, Scambler PJ, Garrick D, Gibbons RJ, Higgs DR, Cristea IM, Urnov FD, Zheng D, Allis CD (2010) Distinct factors control histone variant H3.3 localization at specific genomic regions. Cell 140:678-691

7. Jiao Y, Shi C, Edil BH, de Wilde RF, Klimstra DS, Maitra A, Schulick RD, Tang LH, Wolfgang CL, Choti MA, Velculescu VE, Diaz LA Jr, Vogelstein B, Kinzler KW, Hruban RH, Papadopoulos N (2011) DAXX/ATRX, MEN1, and mTOR pathway genes are frequently altered in pancreatic neuroendocrine tumors. Science 331:1199-1203

8. Tsourlakis MC, Schoop M, Plass C, Huland H, Graefen M, Steuber T, Schlomm T, Simon R, Sauter G, Sirma H, Minner S (2013) Overexpression of the chromatin remodeler death-domainassociated protein in prostate cancer is an independent predictor of early prostate-specific antigen recurrence. Hum Pathol 44:1789-1796

9. Pan WW, Zhou JJ, Liu XM, Xu Y, Guo LJ, Yu C, Shi QH, Fan HY (2013) Death domain-associated protein DAXX promotes ovarian cancer development and chemoresistance. J Biol Chem 288: 13620-13630

10. Tang J, Qu LK, Zhang J, Wang W, Michaelson JS, Degenhardt YY, El-Deiry WS, Yang X (2006) Critical role for Daxx in regulating Mdm2. Nat Cell Biol 8:855-862

11. Song MS, Salmena L, Carracedo A, Egia A, Lo-Coco F, TeruyaFeldstein J, Pandolfi PP (2008) The deubiquitinylation and localization of PTEN are regulated by a HAUSP-PML network. Nature 455:813-817

12. Chen YW, Lin GJ, Chia WT, Lin CK, Chuang YP, Sytwu HK (2009) Triptolide exerts anti-tumor effect on oral cancer and KB cells in vitro and in vivo. Oral Oncol 45:562-568

13. Wu MJ, Jan CI, Tsay YG, Yu YH, Huang CY, Lin SC, Liu CJ, Chen YS, Lo JF, Yu CC (2010) Elimination of head and neck cancer initiating cells through targeting glucose regulated protein 78 signaling. Mol Cancer 9:283-298

14. Michaelson JS, Leder P (2003) RNAi reveals anti-apoptotic and transcriptionally repressive activities of DAXX. J Cell Sci 116: 345-352

15. Nateri AS, Spencer-Dene B, Behrens A (2005) Interaction of phosphorylated c-Jun with TCF4 regulates intestinal cancer development. Nature 437:281-285

16. Chang CC, Naik MT, Huang YS, Jeng JC, Liao PH, Kuo HY, Ho CC, Hsieh YL, Lin CH, Huang NJ, Naik NM, Kung CC, Lin SY, Chen RH, Chang KS, Huang TH, Shih HM (2011) Structural and functional roles of Daxx SIM phosphorylation in SUMO paralogselective binding and apoptosis modulation. Mol Cell 42:62-74

17. Huang YS, Shih HM (2009) Daxx positively modulates beta-catenin/TCF4-mediated transcriptional potential. Biochem Biophys Res Commun 386:762-768

18. Hanken H, Grobe A, Cachovan G, Smeets R, Simon R, Sauter G, Heiland M, Blessmann M (2014) CCND1 amplification and cyclin D1 immunohistochemical expression in head and neck squamous cell carcinomas. Clin Oral Investig 18:269-276

19. Tetsu O, McCormick F (1999) Beta-catenin regulates expression of cyclin D1 in colon carcinoma cells. Nature 398:422-426

20. Zhao Y, Yu D, Li H, Nie P, Zhu Y, Liu S, Zhu M, Fang B (2014) Cyclin D1 overexpression is associated with poor clinicopathological outcome and survival in oral squamous cell carcinoma in Asian populations: insights from a meta-analysis. PLoS One 9:e93210 e93218

21. Kwan PS, Lau CC, Chiu YT, Man C, Liu J, Tang KD, Wong YC, Ling MT (2013) Daxx regulates mitotic progression and prostate cancer predisposition. Carcinogenesis 34:750-759

22. Pan WW, Yi FP, Cao LX, Liu XM, Shen ZF, Bu YQ, Xu Y, Fan HY, Song FZ (2013) DAXX silencing suppresses mouse ovarian surface epithelial cell growth by inducing senescence and DNA damage. Gene 526:287-294

23. da Silva SD, Ferlito A, Takes RP, Brakenhoff RH, Valentin MD, Woolgar JA, Bradford CR, Rodrigo JP, Rinaldo A, Hier MP, Kowalski LP (2011) Advances and applications of oral cancer basic research. Oral Oncol 47:783-791

24. Emelyanov AV, Kovac CR, Sepulveda MA, Birshtein BK (2002) The interaction of Pax5 (BSAP) with Daxx can result in transcriptional activation in B cells. J Biol Chem 277:11156-11164

25. Salomoni P (2013) The PML-interacting protein DAXX: histone loading gets into the picture. Front Oncol 3:152-162 